\title{
Stöver-Blahak, Anke: Sprechen und Vortragen lernen im Fremdsprachenunterricht. Interpretativ, kreativ und ganzheitlich mit Gedichten.
}

\author{
Internationaler Verlag der Wissenschaften Peter Lang, \\ Frankfurt/Main, 2012, 328 Seiten, ISBN \\ print: 978-3-631-62518-7, ISBN eBook: 978-3-653-02419-7

\section{Gerlinde Kempendorff-Hoene}

Ausgerechnet Gedichte - literarische Hochsprache - im Fremdsprachenunterricht; ist das nicht zuviel Information für Fremdsprachler auf einmal? Lenkt es nicht vom eigentlichen Spracherwerb ab, auch noch auf die Interpretation von Gedichten (Rilke, Fried, Hesse...) zu achten und sich über die Bedeutung von Metaphern in einer fremden Sprache Gedanken machen zu müssen? Sich über Versmaß zu informieren und seine eigenen Gefühle in einer Fremdsprache zu artikulieren?

Die Arbeit von Anke Stöver-Blank beweist empirisch mit einer Vielzahl praktischer Belege anhand von Versuchsreihen mit Studierenden in verschiedenen Stufen der Gedichterarbeitung (auch als DVD akribisch aufgearbeitet und dem Buch beigefügt) und mit wissenschaftlicher Penibilität, dass dies ein Gewinn in vielerlei Hinsicht ist.

Nach neuesten Erkenntnissen der Gehirnforschung ist es sogar einem „alten Mann von 80 Jahren möglich, Chinesisch zu lernen, wenn er sich in eine Chinesin verliebt hat" (frei zitiert nach Gerald Hüther). Das heißt, der emotionale Faktor bei Lernprozessen hat bisher zuwenig offizielle Beachtung gefunden. Anke Stöver-Blahak ist es gelungen dies wissenschaftlich nachzuweisen. Die Säule der Sprechwissenschaft/Sprecherziehung - „Ästhetische Kommunikation“ - wird durch das Arbeitsmaterial Gedichte in den Vordergrund gerückt. Die Autorin macht in ihrer Arbeit klar, dass dem bislang zuwenig Aufmerksamkeit gewidmet wird: Sie plädiert für den Ausbau bzw. die Förderung und Intensivierung dieser Fächer an den Hochschulen (dem kann sich die Verfasserin nur anschließen) und zitiert Roland Forster:

Wer über Sprechwissenschaft/Sprecherziehung redet oder publiziert, stößt häufig auf fehlendes Wissen über dieses Fach und seine Gegenstände, erweckt mit seinen Ausführungen immer aber Neugier und Interesse. Dies gilt auch für den Bereich des modernen Fremdsprachenlernens. (5)

In der Arbeit wird zu Recht für die Mehrschichtigkeit des ästhetischen Lernens in der Fremdsprachendidaktik plädiert, greift doch der Studierende laut Stöver-Blahak 
... auf mindestens drei Wissensquellen zurück: Auf seine Heimat- oder Herkunftskultur, Weltwissen und auf das, was er (der Sprecher, d.V.) von der Zielkultur schon weiß. (40)

Und:

Die Beziehung zwischen dem Sprecher und dem Gedicht ist eine ganzheitliche, Elemente von Fremdheit - etwa das Nichtverstehen von Wörtern, das Nichtsprechenkönnen einzelner Lautphänomene aber auch das Wahrnehmen oder gar Nichtverstehen des kulturell Anderen - treten als Hindernisse im Erarbeiten der Sprechfassung auf und werden in diesem Kontext bearbeitet und bestenfalls gelöst. (ibid)

Natürlich könnte man jetzt behaupten, die LernerInnen könnten das an jedem Prosatext auch tun. Was zu beweisen wäre. Aber der verdichtete Text eines Gedichtes in der Hochform der zu lernenden Fremdsprache fördert die hier so bewiesenen Aspekte des Fremdspracherwerbs besonders gut.

Das Argument von Anke Stöver-Blahak, dass es ,inhaltloses Sprechen und emotionsloses Sprechen nicht gibt" (126), erklärt, warum sie Gedichten als Übungstext den Vorzug gibt. Wenn, wie anfänglich nach Gerald Hüther zitiert, der emotionale Aspekt beim Fremdspracherwerb ein viel höherer ist als bisher berücksichtigt wurde, sind Gedichte in ihrer emotionalen Sprachverdichtung dadurch ein ideales Arbeitsmaterial. Das wird auch in den ausführlichen LernerInnenprofilen (233ff) noch einmal deutlich, wenn die Studentin Justyna aussagt, dass sie „dieses mit dem stärksten Verständnis lesen konnte“ und sie „das tiefe Sehnsuchtsgefühl sofort erkannt habe“. Weiter wird ausgeführt:

Den Ausgangspunkt bildet also ein deutlich autobiographischer emotionaler Bezug, ein Inhalt, der aber schon im Auswahlverfahren mit der Umsetzung in Sprechen/Vortragen verbunden wird. (285)

Die Ergebnisse der Studie werden schriftlich und medial ausgewertet. Sie sind nach LernerInnen geordnet, auch in einzelnen Filmsequenzen zu beobachten und in Tabellen und Grafiken gut nachvollziehbar. Die Gliederung in sieben Kapitel ist in ihrer Struktur voll ausgereizt, ein ausführliches Literaturverzeichnis und die DVD im Anhang runden die Arbeit als eine praktische Hilfe für Lehrende im Fremdspracherwerb ab. Und dem Fazit von Anke Stöver-Blahak ist nichts hinzuzusetzen:

Geforscht wird aus der Praxis für die Praxis. (311)

Das zentrale Forschungsergebnis dieser Studie ist, dass Methoden der Ästhetischen Kommunikation im Fremdsprachenunterricht (Deutsch) in studienbegleitenden heterogenen Gruppen an Universitäten sinnvoll eingesetzt werden können. Durch sie kann die festgestellte Lücke zwischen Phonetikunterricht und Präsentationstraining geschlossen werden. (312) 
P.S.: Außer: Dass das möglich ist, setzt die Finanzierung von Schlüsselkompetenzen an den Universitäten voraus. An der Universität Potsdam werden gerade zum kommenden Sommersemester die Mittel für das Schlüsselkompetenzzentrum Studiumplus wieder mal gekürzt, obwohl hohe Anmeldungszahlen von Studierenden und sehr gute Evaluierungen der letzten Jahre die Nachfrage belegen.

P.P.S.: Zum Layout des Buches sei angemerkt, dass es nicht besonders leserfreundlich ist. Die Schriftgröße ist in der angebotenen Textdichte klein, es gibt wenige Absätze, und das Format des Buches erschwert bei einer Seitenzahl von 340 ein Offenliegen an einer bestimmten Seite, auf der man arbeiten möchte. 